\title{
Editorial: Developmental Programming of Metabolic Diseases
}

\author{
Sarah J. Glastras ${ }^{1,2}$, Damaskini Valvi ${ }^{3 *}$ and Amita Bansal ${ }^{4 *}$ \\ ${ }_{1}^{1}$ Department of Diabetes, Metabolism and Endocrinology, and Kolling Institute, Royal North Shore Hospital, Sydney, NSW, \\ Australia, ${ }^{2}$ Sydney Medical School, University of Sydney, NSW, Australia, ${ }^{3}$ Department of Environmental Medicine and \\ Public Health, Icahn School of Medicine at Mount Sinai, New York, NY, United States, ${ }^{4}$ Australian National University (ANU) \\ Medical School, and John Curtin School of Medical Research, College of Health and Medicine, Australian National University, \\ Canberra, ACT, Australia
}

Keywords: developmental programming, metabolism, fructose, vitamin B12, mitochondrial dysfunction, adiposity, metformin, adipocyte precursors

\section{Editorial on the Research Topic \\ Developmental Programming of Metabolism}

\section{OPEN ACCESS}

Edited and reviewed by: James M. Olcese,

Florida State University, United States

${ }^{*}$ Correspondence:

Damaskini Valvi dania.valvi@mssm.edu Amita Bansal amita.bansa@@anu.edu.au

Specialty section: This article was submitted to Translational Endocrinology, a section of the journal Frontiers in Endocrinology

Received: 22 September 2021 Accepted: 05 October 2021 Published: 21 October 2021

Citation:

Glastras SJ, Valvi D and Bansal A (2021) Editorial: Developmental Programming of Metabolic Diseases.

Front. Endocrinol. 12:781361. doi: 10.3389/fendo.2021.781361
Environmental exposures from pre-conception through early postnatal life are associated with adverse consequences for the offspring and may increase the likelihood of developing chronic diseases in adult life, such as type 2 diabetes, obesity, cardiovascular disease, and chronic kidney disease (1-5). In this Research Topic series involving 8 peer-reviewed articles, we focus on maternal factors that can lead to adverse developmental programming of offspring metabolism (Smith et al.; Damron et al.; Behere et al.), consider cellular mechanisms of foetal programming (Pendleton et al.; Ying and Simmons; Poon), discuss the programming effects on various organs specifically liver, skeletal muscle, adipose tissue and the brain (Pendleton et al.; Ying and Simmons; Poon), and draw attention to intervention strategies that may prevent the risk of metabolic disturbance to the offspring (Li et al.; 6).

Maternal exposure to either nutrient excess or deficit can have long-term consequences for offspring's metabolic health. The study by Smith et al. using a novel guinea pig model of maternal high-fructose diet exposure, demonstrates perturbed metabolic outcomes for the offspring, including higher free fatty acids, increased serum uric acid and triglycerides (Smith et al.). In addition Damron et al. observed that maternal high-fat feeding from 4 weeks prior to mating throughout pregnancy in C57BL/6J mice induced sex-specific effects in the offspring, such that male offspring had increased adiposity, impaired glucose tolerance and reduced pancreatic insulin secretion (Damron et al.). Similar metabolic perturbations have been previously described in the offspring whose mothers were exposed to high-fat diet or environmental chemicals during pregnancy (7-10). Furthermore, Behere et al, in a systematic review of studies conducted in India, observed high prevalence of vitamin B12 deficiency in women in India during pregnancy with potential adverse outcomes for the offspring's metabolic health (Behere et al.). Their systematic review supports the inclusion of vitamin B12 to existing nutritional programs in India for extended benefits on outcomes in pregnancy and offspring health besides control of anaemia. Together these studies indicate that maternal exposure to a sub-optimal environment can impair offspring's metabolic health in the long-term and highlight a need for interventions around pregnancy, which will prove beneficial in curtailing these long-term metabolic sequelae.

The mechanisms underpinning the association of maternal factors with increased susceptibility of the offspring to develop obesity, type 2 diabetes, heart disease and other components of metabolic syndrome in adulthood are not fully elucidated and increasingly investigated. Inflammation, oxidative 
stress, mitochondrial biogenesis, lipid metabolism and placental function are regarded as key regulators of foetal programming (11-13). Pendleton et al. reviewed the evidence that intrauterine growth restriction induces placental insufficiency, which has profound effects on the developing foetus, with metabolic dysfunction seen in skeletal muscle and liver (Pendleton et al.). Similar metabolic dysfunction following intrauterine growth restriction has been described previously in pancreatic islets also $(14,15)$. Specifically, these are linked to 10-maladaptive mitochondrial changes leading to impaired mitophagy (Pendleton et al.). Mitochondrial dysfunction is implicated in a wide variety of chronic metabolic diseases including but not limited to diabetic kidney disease, obesity and type 2 diabetes $(13,16)$. Simmons and Ying reviewed the latest evidence from experimental models about adipocyte precursors lineage and regulation mechanisms during development and in adulthood. Their review summarize important differences in adipogenesis mechanism during development compared to adult adipogenesis and underscores the need for future investigation on developmental adipocyte precursors in humans and their role in the developmental origins of health and disease (Ying and Simmons). In the review by Poon, the evidence of the impact of maternal obesity on hypothalamic neurocircuitry highlights that prenatal high-fat diet exposure increases neurogenesis of neuropeptide systems in offspring brain and influences behaviour with respect to food seeking behaviour (Poon). The review specifically discussed the role of inflammatory mediators, chemokines, in this process, illustrating how upregulation of chemokines following a high fat diet intake impacts brain neurochemistry in offspring, thereby mediating the neurogenic effects of a high fat diet on hypothalamic peptide neurons (Poon). Taken together, we are gaining greater understanding of the mechanisms underpinning developmental programming of metabolic disease and reputed players, such as the mitochondria and immune system are emerging to play a central role.

Preventing adverse programming at preconception is perhaps the most attractive approach that promotes metabolic health starting early in life. In this Research Topic series, $\mathrm{Xu}$ et al. discuss the potential role of metformin therapy during gestation, and $\mathrm{Li}$ et al. investigate the role of low dose aspirin to prevent kidney damage in LPS-induced preeclampsia, discovering that aspirin inhibits the WNT5A and NF-kB signalling pathway. In the context of maternal overnutrition, low dose hydralazine, a demethylating agent safe in pregnancy has also demonstrated reduced renal fibrosis in rodent offspring $(17,18)$. Ongoing future research to establish safe and effective treatments that can be safely

\section{REFERENCES}

1. Hoffman DJ, Powell TL, Barrett ES, Hardy DB. Developmental Origins of Metabolic Diseases. Physiol Rev (2021) 101(3):739-95. doi: 10.1152/physrev.00002.2020

2. Stephenson J, Heslehurst N, Hall J, Schoenaker DA, Hutchinson J, Cade JE, et al. Before the Beginning: Nutrition and Lifestyle in the Preconception Period and its Importance for Future Health. Lancet (2018) 391(10132):183041. doi: 10.1016/S0140-6736(18)30311-8

3. Glastras SJ, Tsang M, Teh R, Chen H, McGrath RT, Zaky AA, et al. Maternal Obesity Promotes Diabetic Nephropathy in Rodent Offspring. Sci Rep (2016) 6:27769. doi: 10.1038/srep27769 administered before or during pregnancy are urgently needed to prevent adverse developmental programming of the foetus and improve the long-term health of the offspring. Given our knowledge that postnatal high-fat diet feeding significantly amplifies the adverse metabolic effect of maternal obesity (19), pharmacological strategies to mitigate neurological and psychological drive towards food seeking behaviour are needed. In the past decade, there have been significant advances in pharmacotherapy for weight reduction which include glucose-like peptide-1 (GLP-1) receptor agonists, utilised previously in the offspring of a mouse model of maternal obesity to rescue offspring metabolic dysfunction (20). More recently, tesofensine, a novel monoamine reuptake inhibitor that inhibits both norepinephrine, serotonin, and dopamine reuptake function, has been developed as a promising new treatment for obesity (21). There remains a paucity of evidence as to the benefit to mother and the offspring of maternal weight reduction prior to conception in females with obesity.

Though genetic predisposition and postnatal environmental exposures are important aetiological factors for the development of chronic disease over the life-course, maternal health and in utero environmental exposures can perturbate metabolic programming in the foetus through direct mechanisms with long-lasting adverse metabolic effects over the lifespan. The preconception and pregnancy windows offer unique opportunities for interventions that promote optimal health for both the mother and the offspring. Findings summarized in this Special Issue provide novel insights into the mechanisms underlying the developmental origins of metabolic diseases and highlight treatments that prevent metabolic risks starting early in life. These findings will inform the design of more effective interventions focused on the prevention of environmental triggers, maternal nutrition and/or potential therapeutic targets.

\section{AUTHOR CONTRIBUTIONS}

SG, DV, AB conceptualized, wrote, edited, and approved the final version of the manuscript. All authors contributed to the article and approved the submitted version.

\section{ACKNOWLEDGMENTS}

We thank all the authors who contributed their work to this Research Topic and all the reviewers who generously reviewed the manuscripts and provided valuable comments to improve scientific content and clarity.

4. Valvi D, Højlund K, Coull BA, Nielsen F, Weihe P, Grandjean P. Life-Course Exposure to Perfluoroalkyl Substances in Relation to Markers of Glucose Homeostasis in Early Adulthood. J Clin Endocrinol Metab (2021) 106 (8):2495-504. doi: 10.1210/clinem/dgab267

5. Bansal A, Li C, Xin F, Duemler A, Li W, Rashid C, et al. Transgenerational Effects of Maternal Bisphenol: A Exposure on Offspring Metabolic Health. J Dev Orig Health Dis (2019) 10(2):164-75. doi: 10.1017/ S2040174418000764

6. Wu M, Xu H, Liu J, Tan X, Wan S, Guo M, et al. Metformin and Fibrosis: A Review of Existing Evidence and Mechanisms. J Diabetes Res (2021) 2021:6673525. doi: 10.1155/2021/6673525 
7. Glastras SJ, Chen H, Pollock CA, Saad S. Maternal Obesity Increases the Risk of Metabolic Disease and Impacts Renal Health in Offspring. Biosci Rep (2018) 38(2):BSR20180050. doi: 10.1042/BSR20180050

8. Bansal A, Rashid C, Xin F, Li C, Polyak E, Duemler A, et al. Sex- and DoseSpecific Effects of Maternal Bisphenol A Exposure on Pancreatic Islets of First- and Second-Generation Adult Mice Offspring. Environ Health Perspect (2017) 125(9):097022. doi: 10.1289/EHP1674

9. Valvi D, Casas M, Mendez D, Ballesteros-Gómez MA, Luque A, Rubio N, et al. Prenatal Bisphenol a Urine Concentrations and Early Rapid Growth and Overweight Risk in the Offspring. Epidemiology (2013) p:791-9. doi: 10.1097/ EDE.0b013e3182a67822

10. Glastras SJ, Rashid C, Xin F, Li C, Polyak E, Duemler A, et al. Mouse Models of Diabetes, Obesity and Related Kidney Disease. PLoS One (2016) 11(8): e0162131. doi: 10.1371/journal.pone.0162131

11. Bansal A, Henao-Mejia J, Simmons RA. Immune System: An Emerging Player in Mediating Effects of Endocrine Disruptors on Metabolic Health. Endocrinology (2018) 159(1):32-45. doi: 10.1210/en.2017-00882

12. Elshenawy S, Pinney SE, Stuart T, Doulias PT, Zura G, Parry S, et al. The Metabolomic Signature of the Placenta in Spontaneous Preterm Birth. Int $J$ Mol Sci (2020) 21(3):1043. doi: 10.3390/ijms21031043

13. Bansal A, Rashid C, Simmons RA. Impact of Fetal Programming on Mitochondrial Function and Susceptibility to Obesity and Type 2 Diabetes. In: Mitochondria in Obesity and Type 2 Diabetes. Elsevier (2019). p. 325-45. Available at: https://www.sciencedirect.com/science/article/pii/B97801281 17521000146

14. Gatford KL, Simmons RA. Prenatal Programming of Insulin Secretion in Intrauterine Growth Restriction. Clin Obstet Gynecol (2013) 56(3):520-8. doi: 10.1097/GRF.0b013e31829e5b29

15. Rashid CS, Lien YC, Bansal A, Jaeckle-Santos LJ, Li C, Won KJ, et al. Transcriptomic Analysis Reveals Novel Mechanisms Mediating Islet Dysfunction in the Intrauterine Growth-Restricted Rat. Endocrinology (2018) 159(2):1035-49. doi: 10.1210/en.2017-00888

16. Huang C, Yi H, Shi Y, Cao Q, Shi Y, Cheng D, et al. KCa3.1 Mediates Dysregulation of Mitochondrial Quality Control in Diabetic Kidney Disease. Front Cell Dev Biol (2021) 9:573814. doi: 10.3389/fcell.2021.573814

17. Larkin BP, Glastras SJ, Chen H, Pollock CA, Saad S. DNA Methylation and the Potential Role of Demethylating Agents in Prevention of Progressive
Chronic Kidney Disease. FASEB J (2018) 32(10):5215-26. doi: 10.1096/ fj.201800205R

18. Larkin BP, Saad S, Glastras SJ, Nguyen LT, Hou M, Chen H, et al. Low-Dose Hydralazine During Gestation Reduces Renal Fibrosis in Rodent Offspring Exposed to Maternal High Fat Diet. PLoS One (2021) 16(3):e0248854. doi: 10.1371/journal.pone. 0248854

19. Glastras SJ, Chen H, Tsang M, Teh R, McGrath RT, Zaky A, et al. The Renal Consequences of Maternal Obesity in Offspring are Overwhelmed by Postnatal High Fat Diet. PLoS One (2017) 12(2):e0172644. doi: 10.1371/ journal.pone. 0172644

20. Glastras SJ, Chen H, McGrath RT, Zaky AA, Gill AJ, Pollock CA, et al. Effect of GLP-1 Receptor Activation on Offspring Kidney Health in a Rat Model of Maternal Obesity. Sci Rep (2016) 6:23525. doi: 10.1038/srep23525

21. Axel AMD, Mikkelsen JD, Hansen HH. Tesofensine, a Novel Triple Monoamine Reuptake Inhibitor, Induces Appetite Suppression by Indirect Stimulation of $\alpha 1$ Adrenoceptor and Dopamine D1 Receptor Pathways in the Diet-Induced Obese Rat. Neuropsychopharmacology (2010) 35(7):1464-76. doi: 10.1038/npp.2010.16

Conflict of Interest: The authors declare that the research was conducted in the absence of any commercial or financial relationships that could be construed as a potential conflict of interest.

Publisher's Note: All claims expressed in this article are solely those of the authors and do not necessarily represent those of their affiliated organizations, or those of the publisher, the editors and the reviewers. Any product that may be evaluated in this article, or claim that may be made by its manufacturer, is not guaranteed or endorsed by the publisher.

Copyright (c) 2021 Glastras, Valvi and Bansal. This is an open-access article distributed under the terms of the Creative Commons Attribution License (CC BY). The use, distribution or reproduction in other forums is permitted, provided the original author(s) and the copyright owner(s) are credited and that the original publication in this journal is cited, in accordance with accepted academic practice. No use, distribution or reproduction is permitted which does not comply with these terms. 